\title{
Design of Delay Locked Loop with Frequency Division Technique
}

\author{
Wenyuan $\mathrm{Li}^{+}$and Yurong Cao \\ Institute of RF-\&OE-ICs, Southeast University, Nanjing 210096, China
}

\begin{abstract}
Design of delay locked loop (DLL) with frequency division technique is presented. The proposed pseudo-differential delay cell increases the input clock frequency. To avoid the false locking or harmonic locking problem, a simple start-control signal is added. The DLL design are implemented by IBM $0.13 \mu \mathrm{m}$ CMOS process. The static phase error of the DLL is $2.6 \mathrm{ps}$ at $3 \mathrm{GHzand} 8.5 \mathrm{ps}$ at $5 \mathrm{GHz}$, respectively. The peak-to-peak jitter values at $3 \mathrm{GHz}$ and $5 \mathrm{GHz}$ are $3.3 \mathrm{ps}$ and $1.7 \mathrm{ps}$. The supply voltage is $1.2 \mathrm{~V}$.
\end{abstract}

Keywords: delay locked loop, charge pump, phase frequency detector, delay cell, frequency division.

\section{Introduction}

In the recent years, the delay locked loop (DLL)is used for synchronization, clock generation [1], clock deskewing, and data recovery [2]. Compared with phase locked loop (PLL) [3], DLL has better jitter performance because of no jitter accumulation at the end of the voltage-controlled delay line.

The frequency division technique is adopted to not only relax the maximum operation frequency, but also to keep the total delay time equal to one clock cycle of the input clock [4]. To avoid the false locking and harmonic locking problem, a start-control signal is added to the DLL circuit.

\section{Circuit Description}

The conventional DLL is composed of phase detector (PD), charge pump (CP), voltage-controlled delay line(VCDL), loop filter (LF).The DLL is locked until the delay time of VCDL is equal to one clock cycle of the input signal. However, if the initial delay time of VCDL is shorter than $0.5 \mathrm{~T}_{\mathrm{REF}}$ or longer than $1.5 \mathrm{~T}_{\mathrm{REF}}$, the DLL will fail to lock or harmonic locking will occur.

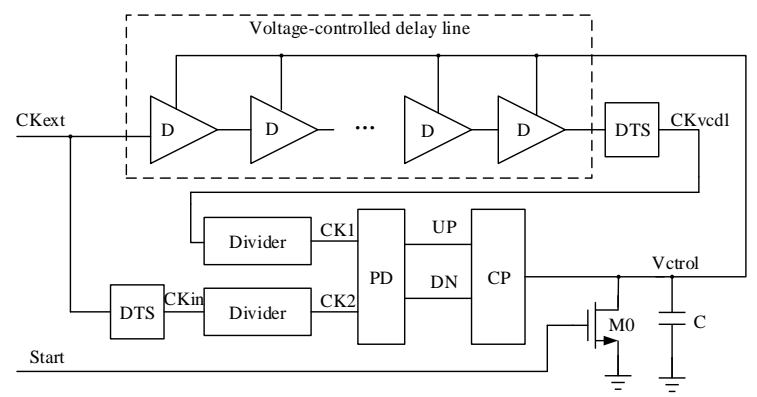

Fig. 1: Proposed DLL architecture.

The proposed DLL architecture is shown in Fig. 1. It consists of PD, CP, VCDL, frequency dividers, and differential-to-single (DTS) circuits. Here, a start-control signal and NMOS transistor M0 are used to ensure the initial delay longer than $0.5 \mathrm{~T}_{\mathrm{REF}}$. When the DLL begins to operate, the start-signal is high and the control voltage is set to minimum so that the total delay is minimum. Thus, the false locking and harmonic locking problem is solved. The total delay time is the delay between CKin and CKvcdl.

+ Corresponding author. Tel.: + 86-258-3793303-8109; fax: +86-25-63792882.

E-mail address:1wy555@seu.edu.cn. 


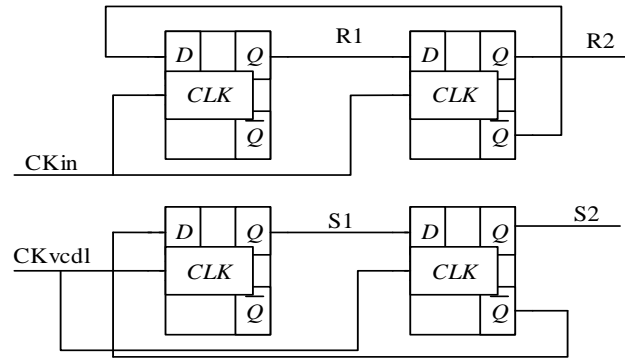

Fig. 2: Two divide-by-4 dividers.

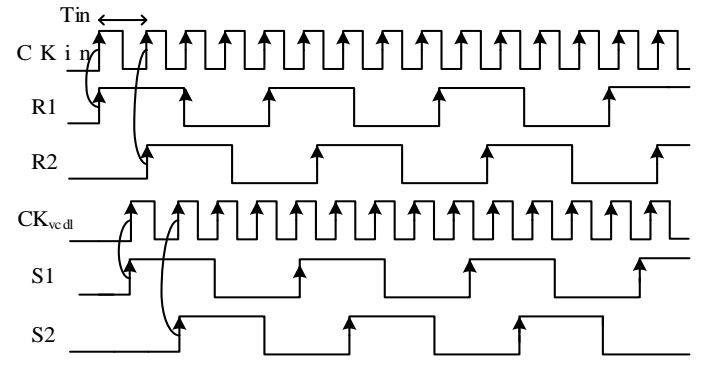

Fig. 3: Timing diagram of the proposed divider.

\subsection{Frequency divider}

Fig. 2 shows the two proposed divide-by- 4 dividers and its simplified timing diagram is also given in Fig.3. They generate two different phase outputs R1, R2 and S1, S2, respectively. From the diagram, R2 is a clock period delay of R1, and the same is between S1 and S2. Comparing the phase difference of R2 and S1, it is found that the difference of them is just right zero in DLL's steady state. In other words, the delay time between CKin and CKvcdl is equal to one clock cycle of CKin.

\subsection{Phase frequency detector}

Although the division technique is adopted to relax the operation frequency of the PFD, it is still necessary to improve the operating speed of it. Meanwhile the dead-zone problem of the PFD [5] should be considered to reduce the static phase error. So in this work, an edge trigger style PFD is proposed. In the PFD, D Flip-Flop adopts TSPC (True Single Phase Clock) DFF to improve the operating frequency of the PFD.

\subsection{Charge pump}

Fig. 4 shows the schematic of proposed current-splitting CP. When UP is high and DN is low, the tail current only passes through M1 and M3 to discharge the subsequent capacitor $\mathrm{C}$. The case reverses if UP is low and DN is high. When the signal UP is the same of DN, the charging current Iout should be zero. To reduce the current mismatch, I0should be set in a smaller value.

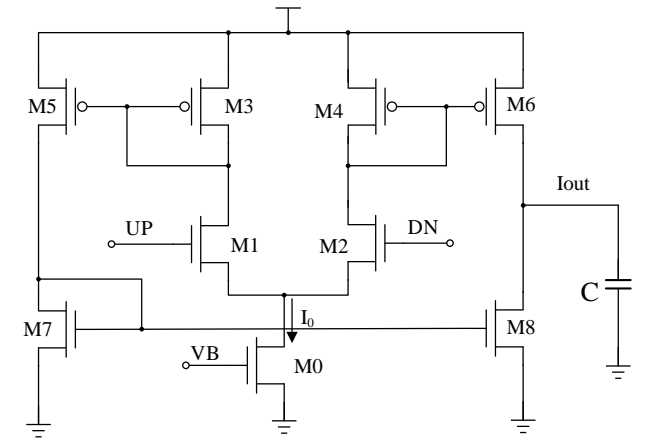

Fig. 4: Current-Splitting CP.

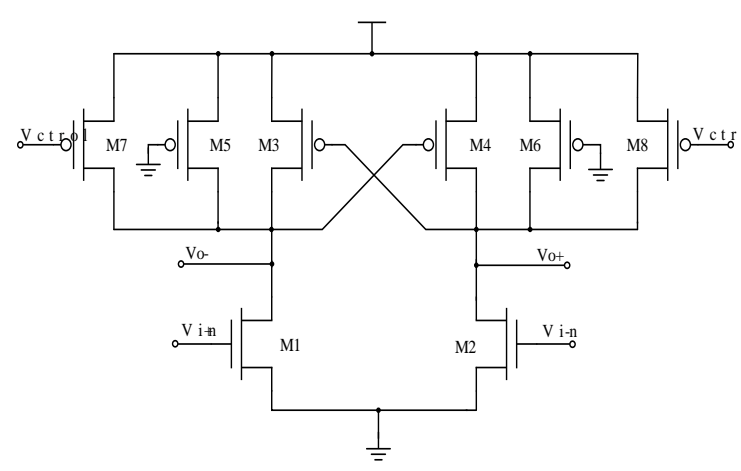

Fig. 5: Proposed voltage-controlled delay cell.

\subsection{Voltage-controlled delay cell}

Fig. 5 shows a pseudo-differential delay cell. M3 and M4 are adopted to speed up the transition of the input signal due to positive feedback. M5, M6 is used to increase charging speed and improve driving ability. M7, M8 is adopted to adjust the delay by changing the value of Vctrol.

\section{Simulation Results}

The DLL has been designed in $0.13 \mu \mathrm{m}$ CMOS process by IBM. Fig. 6 shows the chip layout, which occupies $0.49 \times 0.69 \mathrm{~mm}^{2}$. Fig. 7 shows the delay curve of the VCDL. The input frequency $3-5 \mathrm{GHz}$ can be realized. The starting delay time is $181 \mathrm{ps}$ which is more than $0.5 \mathrm{~T}_{\mathrm{REF}}$ and less than $1.5 \mathrm{~T}_{\mathrm{REF}}$, so the locking problem has been solved. The static phase error is 8.5ps@5GHz and2.6ps@3GHz. Fig. 8 and Fig. 9 show that the control voltage becomes invariant after around 300ns@5GHz and around 500ns@3GHz. Fig. 10 and Fig. 11 show the eye diagram of the DLL. The peak-to-peak jitter of the DLL is $1.7 \mathrm{ps} @ 5 \mathrm{GHz}$ and 3.3ps @3GHz, respectively. 


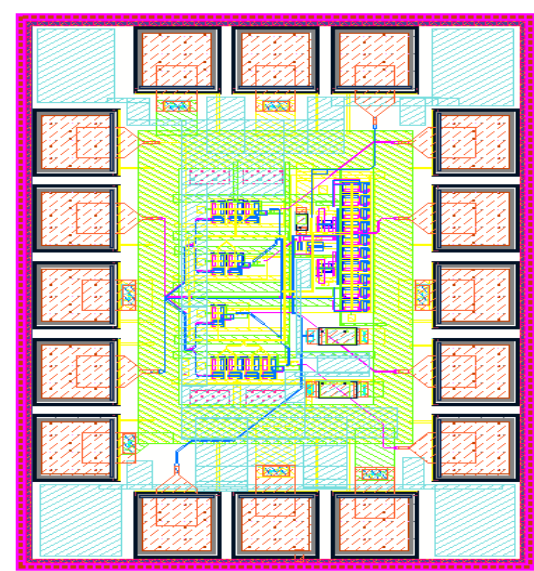

Fig. 6: Layout of the proposed DLL.

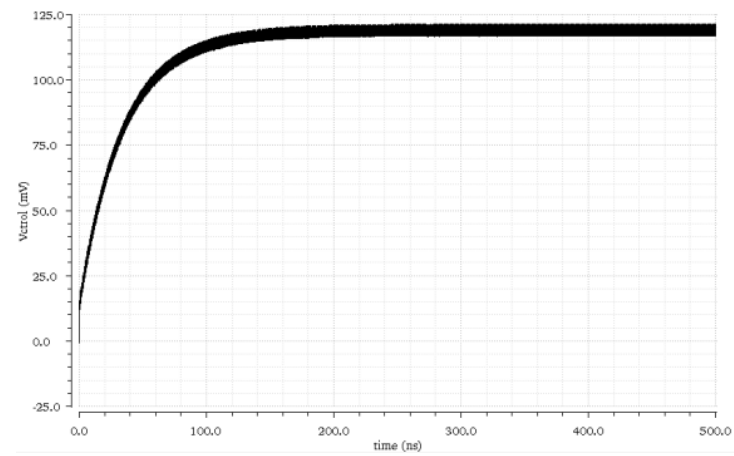

Fig. 8: The control voltage curve@5GHz.

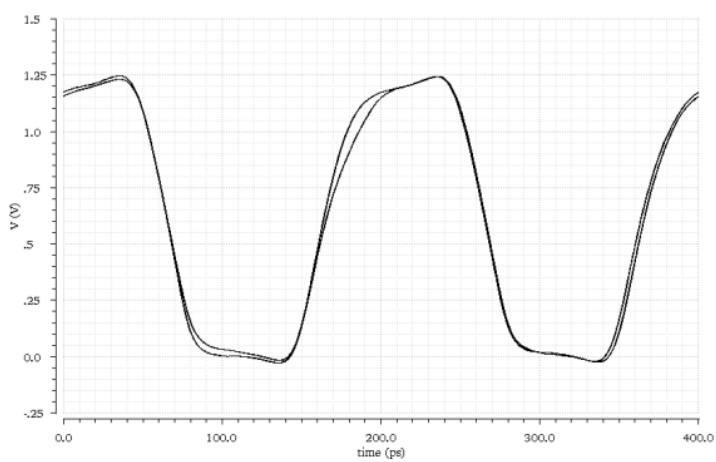

Fig. 10: Peak-to-peak jitter@5GHz.

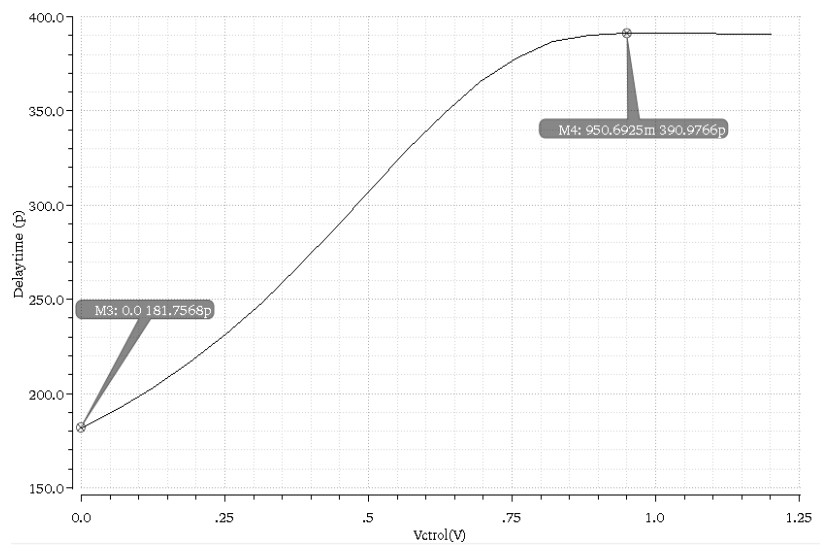

Fig. 7: Delay curve of the VCDL.

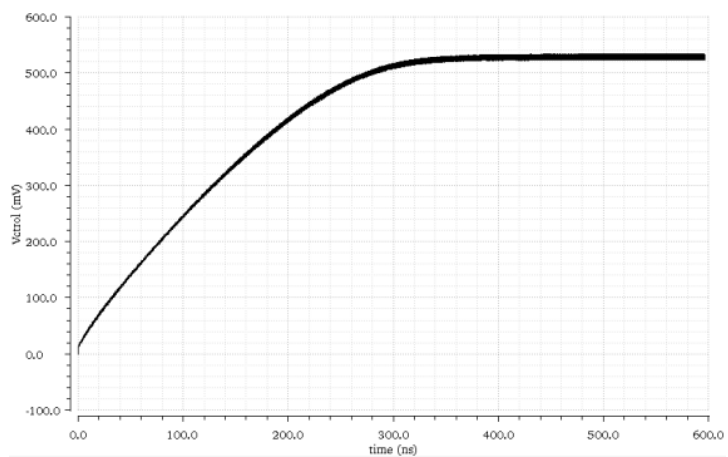

Fig. 9: The control voltage curve@3GHz.

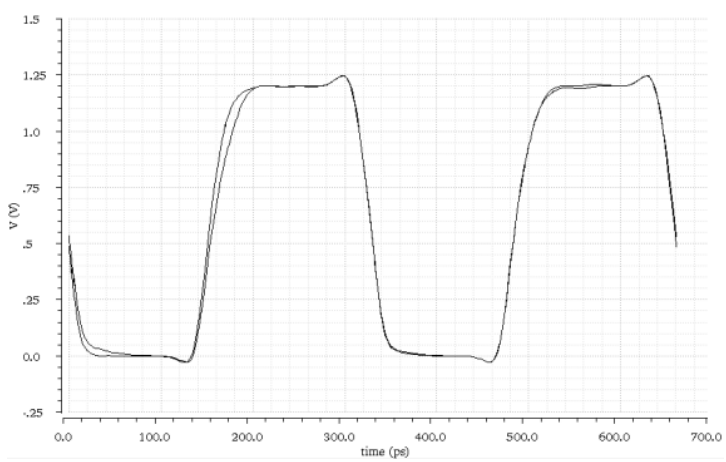

Fig. 11: Peak-to-peak jitter@3GHz.

Compared with the previous DLLs, the DLL in this paper achieve high input frequency and low jitter performance. The comparison results show in Table I. The area of this DLL Chip contains the pad areas.

Table I: Performance Comparison with State-of-the-Art Temperature Sensors

\begin{tabular}{|c|c|c|c|c|}
\hline Parameter & This work & {$[6]$} & {$[7]$} & {$[8]$} \\
\hline Process & $0.13 \mu \mathrm{m}$ & $0.13 \mu \mathrm{m}$ & $0.15 \mu \mathrm{m}$ & $0.18 \mu \mathrm{m}$ \\
\hline Supply & $1.2 \mathrm{~V}$ & $1.2 \mathrm{~V}$ & $1.6 \mathrm{~V}$ & $1.8 \mathrm{~V}$ \\
\hline Operation range & $3-5 \mathrm{GHz}$ & $0.5-5 \mathrm{GHz}$ & $2.1-3.5 \mathrm{GHz}$ & $0.25-2 \mathrm{GHz}$ \\
\hline Peak-to-peak jitter & $1.7 \mathrm{ps} @ 5 \mathrm{GHz}$ & $8 \mathrm{ps} @ 5 \mathrm{GHz}$ & $40 \mathrm{ps} @ 3.5 \mathrm{GHz}$ & $20 \mathrm{ps} @ 2 \mathrm{GHz}$ \\
\hline Area & $0.338 \mathrm{~mm}^{2}$ & $0.107 \mathrm{~mm}^{2}$ & $0.136 \mathrm{~mm}^{2}$ & $0.046 \mathrm{~mm}^{2}$ \\
\hline
\end{tabular}

\section{Conclusion}

In this brief, the proposed DLL achieves high input frequency with frequency division technique, which ensures the DLL locked in one clock cycle and effectively decrease the numbers of the delay cell. The proposed start signal is used to solve the false locking and harmonic locking problem of the conventional DLL. 


\section{Acknowledgements}

We are sincerely thankful for the support from the Project Funded by the Priority Academic Program Development of Jiangsu Higher Education Institutions (PAPD, No.1104007003), Natural Science Foundation of China (No.61471119) and Topnotch Academic Programs Project of Jiangsu Higher Education Institutions (TAPP) PPZY2015A035.

\section{References:}

[1] K. Ryu, J. Jung, D. H. Jung, J. H. Kim, and S. O. Jung, "High-Speed, Low-Power, and Highly Reliable Frequency Multiplier for DLL-Based Clock Generator," IEEE Transactions on Very Large Scale Integration (VLSI) Systems, vol. 24, pp. 1484-1492, 2015.

[2] Y. Liu, Y. Wang, S. Jia, G. Zhang, and X. Zhang, "A 6.25Gb/s CMOS clock and data recovery DLL with anti-harmonic lock," in Electron Devices and Solid-State Circuits (EDSSC), 2014 IEEE International Conference on Chengdu: IEEE, 2014, pp. 1-2.

[3] H. Guo and T. Kwasniewski, "A DLL fractional M/N frequency synthesizer," in Electrical and Computer Engineering (CCECE), 2015 IEEE 28th Canadian Conference on Halifax, NS: IEEE, 2015, pp. 114-117.

[4] C. N. Chuang and S. I. Liu, "A 40GHz DLL-Based Clock Generator in 90nm CMOS Technology," in Solid-State Circuits Conference, 2007. ISSCC 2007. Digest of Technical Papers. IEEE International San Francisco, CA: IEEE, 2007, pp. 178-595.

[5] W. H. Chen, M. E. Inerowicz and B. Jung, "Phase Frequency Detector With Minimal Blind Zone for Fast Frequency Acquisition," Circuits \& Systems II Express Briefs IEEE Transactions on, vol. 57, pp. 936-940, 2010.

[6] C. N. Chuang and S. I. Liu, "A 0.5-5-GHz Wide-Range Multiphase DLL With a Calibrated Charge Pump," IEEE Transactions on Circuits and Systems II: Express Briefs, vol. 54, pp. 939-943, 2007.

[7] A. Alvandpour, R. K. Krishnamurthy, D. Eckerbert, S. Apperson, B. Bloechel, and S. Borkar, "A 3.5GHz 32mW 150nm multiphase clock generator for high-performance microprocessors," in Solid-State Circuits Conference, 2003. Digest of Technical Papers. ISSCC. 2003 IEEE International San Francisco, CA, USA: IEEE, 2003, pp. $112-482$ vol.1.

[8] B. Kim and L. Kim, "A 250MHz-2GHz wide range delay-locked loop," in Custom Integrated Circuits Conference, 2004. Proceedings of the IEEE 2004: IEEE, 2004, pp. 139-142. 